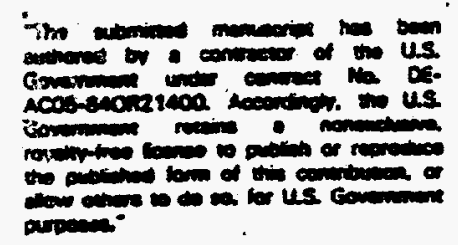

\title{
THREE-DIMENSIONAL ANTENNA MODELS FOR FUSION EXPERIMENTS
}

\author{
M.D. Carter ${ }^{\dagger}$, C.Y. Wang ${ }^{\dagger}$, J.T. Hogan ${ }^{\dagger}$, J.H. Harris ${ }^{\dagger}$, D.J. Hoffman ${ }^{\dagger}$, \\ D.A. Rasmussen ${ }^{\dagger}$, P.M. Ryan ${ }^{\dagger}$, D.S. Stallings ${ }^{\dagger}$, D. B. Batchelor ${ }^{\dagger}$, \\ B. Beaumont ${ }^{\ddagger}$, T. Hutter ${ }^{\ddagger}$, B. Saoutic ${ }^{\ddagger}$, \\ ${ }^{\dagger}$ Oak Ridge National Laboratory, Oak Ridge, TN 37831-8071 USA \\ ${ }^{\ddagger}$ Association Euratom-CEA, Départment de Recherche sur la Fusion Contrôlée, \\ Centre d'Etudes de Cadarache, 13108 Saint Paul Lez Durance, CEDEX FRANCE
}

\begin{abstract}
The development of the RANT3D code has permitted the systematic study of the effect of three-dimensional structures on the launched power spectrum for antennas in the ion cyclotron range of frequencies. The code allows the septa between current straps to be modeled with arbitrary heights and permits the antenna to interact with other structures in the tokamak. In this paper we present comparisons of calculated loading with the Tokamak Fusion Test Reactor and Tore Supra experiments, demonstrate the effects on loading caused by positioning uncertainties for an antenna in Tore Supra, and show electric field patterns near the Tore Supra antenna. A poloidal component in the static magnetic field for the plasma response is included in the near-field calculations using the warm plasma code, GLOSI. Preliminary estimates for the heat flux on the bumper limiters during typical operation in Tore Supra are also presented.
\end{abstract}

\section{INTRODUCTION}

The RANT3D 1 code solves Maxwell's equations in vacuum for an arbitrary number of connected waveguide sections. Perfectly conducting boundary conditions at the walls of the waveguides are satisfied by the basis sets that are used in a Fourier expansion for the fields. Source terms are introduced by specifying the currents on current straps within these waveguides. A large, dense matrix results from equating the tangential electric and magnetic fields at the interface between each of the waveguide sections. The plasma is introduced as a boundary condition by using a generalized impedance matrix ${ }^{2}$. Two codes are used to generate the impedance matrices for the work presented here; both retaining the poloidal and toroidal polarizations for the fields. The first code, PLASMAIMP ${ }^{3}$, is computationally expedient and considers a cold plasma with outgoing boundary conditions near the location of the peak in the density profile. The second code, GLOSI ${ }^{4}$, introduces warm plasma and a sheared poloidal component for the static magnetic field. The loading for the fast wave is nearly the same using either code. However, the near-field patterns for the parallel component of the electric field can be significantly different when a poloidal component is added to the static magnetic field.

\section{LOADING RESULTS}

RANT3D loading resuits agree with experimental measurements of the Tokamak Fusion Test Reactor (TFTR) Bay-M antenna using density profiles measured by reflectometry in a cold plasma calculation 1 . Similar agreement has been found in Tore Supra as shown in Fig.1. This shot is of interest because a loading imbalance was observed between the two straps. Such an imbalance is not expected for a perfectly aligned antenna with dipole phasing. The results in Fig. 1 show the range of loading that can be expected because of a 5-mm difference in spacing between the plasma and the left- and right-hand current straps for dipole phasing. The average value of the calculated loading is in reasonable agreement with the measurements. The range caused by the misalignment is significant but is not large

* Research sponsored by the Office of Fusion Energy, U.S. Department of Energy, under contract DE-AC0584OR21400 with Martin Marietta Energy Systems, Inc. 


\section{DISCLAIMER}

This report was prepared as an account of work sponsored by an agency of the United States Government. Neither the United States Government nor any agency thereof, nor any of their employees, makes any warranty, express or implied, or assumes any legal liability or responsibility for the accuracy, completeness, or usefulness of any information, apparatus, product, or process disclosed, or represents that its use would not infringe privately owned rights. Reference herein to any specific commercial product, process, or service by trade name, trademark, manufacturer, or otherwise does not necessarily constitute or imply its endorsement, recommendation, or favoring by the United States Government or any agency thereof. The views and opinions of authors expressed herein do not necessarily state or reflect those of the United States Government or any agency thereof. 


\section{DISCLAIMER}

Portions of this document may be illegible in electronic image products. Images are produced from the best available original document. 


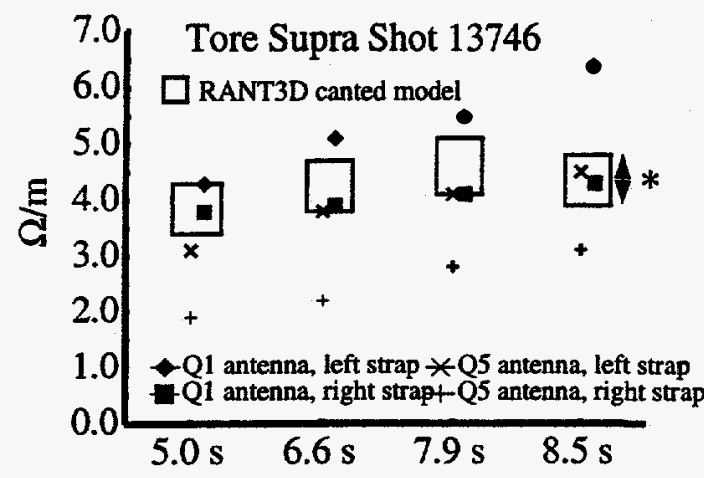

FIGURE 1 Model agrees with measurement.

*Range caused by misalignment. enough to completely account for the imbalance. The uncertainty in the phasing of the current straps is $\sim 20^{\circ}$, but again this uncertainty alone can only partially account for the imbalance. Uncertainty in the measured capacitor settings also results in errors for the loading measurements. It is likely that a combination of these effects is responsible for the imbalance observed in this shot.

\section{NEAR-FIELD RESULTS}

The heat flux on the outside of the frame is important in the Tore Supra experiments. A radio-frequency (RF) rectification model ${ }^{5}$ for the heat flux requires a complete understanding of the RF fields and plasma currents in the vicinity of the bumper limiters as well as the connection of static magnetic field lines to other parts of the tokamak. The RANT3D model presented here does not provide a complete understanding but can provide some insight into the problem. Four phenomena that are notably absent from the model are: 1) The bumpers are actually immersed in plasma rather than vacuum, and the fields may be high enough to cause the RF sheath to arc. Arcing at the sheath would substantially lower the parallel field by allowing a current to flow. 2) Helmholtz's theorem has not been used to separate the electric fields into longitudinal (curl free) and transverse (divergence free) components. The plasma may only be able to rectify the longitudinal component. 3) The Faraday screen model in RANT3D reduces the radial component of the electric field between the current straps and the plasma that is caused by charge on the current straps, but does not locally short the parallel electric field. The plasma surface itself carries currents that reduce this parallel field, however, these plasma currents may be carried into the bumper region affecting the fields at the bumper. 4) Damping caused by electron-neutral collisions. A thorough study of the numerical resolution in RANT3D is also needed.

In the RANT3D model, the assumption that the bumper is in vacuum causes an equivalent voltage $\equiv \mathrm{E} \cdot \mathrm{dl}$ that is too large to be physically reasonable; however, if we assume that the electric field at the plasma surface is comparable to that at a bumper limiter with plasma $1 \mathrm{~mm}$ away, then more reasonable results are obtained. Based on this assumption, two methods for estimating the heat flux from the RANT3D results are presented below. In both cases, the density at the bumper is taken from the reflectometer data to be $3 \times 10^{18} \mathrm{~m}^{-3}$ and the electron temperature is assumed to be $30 \mathrm{eV}$ yielding an incident deuterium flux at the bumper of $c_{s} n_{i} \cong$ $10^{23} \mathrm{~s}^{-1} \mathrm{~m}^{-2}$ where $c_{s}$ is the ion sound speed.

In Fig. 2, the toroidal component of the RF electric field at the plasma surface is shown in real space. A poloidal component is added to the static magnetic field such that the angle of the field line relative to the current strap is $6^{\circ}$. The plasma parameters are taken from the Tore Supra data used in Fig. 1 at $t=5.0 \mathrm{~s}$. The fast wave loading was reduced by $15 \%$ when the magnetic field angle was introduced. Also, the introduction of an angle for the magnetic field significantly changes the pattern of the toroidal component of the electric field.

Assuming that only the longitudinal component of the field is rectified and that the toroidal component of $\mathrm{E}$ is the dominant longitudinal component, then Fig. 2 
gives an estimate for $\int \mathrm{E} \cdot \mathrm{dl} \cong 100 \mathrm{~V}$ for $2 \mathrm{MW}$ of $\mathrm{RF}$ power with dipole phasing. A floating potential of -50 to $60 \mathrm{~V}$ has been measured with Langmuir probes near the antenna for dipole phasing. The case with monopole phasing shows stronger fields near the bottom corners of the antenna than at the midplane such that $\mathrm{JE} \cdot \mathrm{dl} \cong$ $400 \mathrm{~V}$ near the midplane and $\sim 600 \mathrm{~V}$ near the corners. The amplitude of the electric field at the bumper limiter is comparable for both the monopole and dipole phasing, but the scale length is much longer in the monopole case. Combined with the particle flux estimate above, these fields give $\sim 100 \mathrm{~V} \times 10^{23} \mathrm{~s}^{-1} \mathrm{~m}^{-2} \times 1.6 \times 10^{-19} \mathrm{~J} / \mathrm{eV} \cong$ $1.6 \mathrm{MW} / \mathrm{m}^{2}$ at the leading edge of the bumper limiter in Tore Supra for dipole operation. An experimentally measured heat flux comparable with this number has

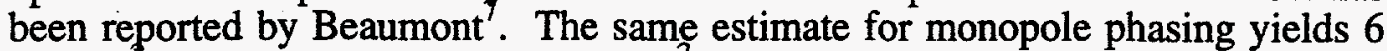
$\mathrm{MW} / \mathrm{m}^{2}$ on the midplane and $10 \mathrm{MW} / \mathrm{m}^{2}$ near the corners.
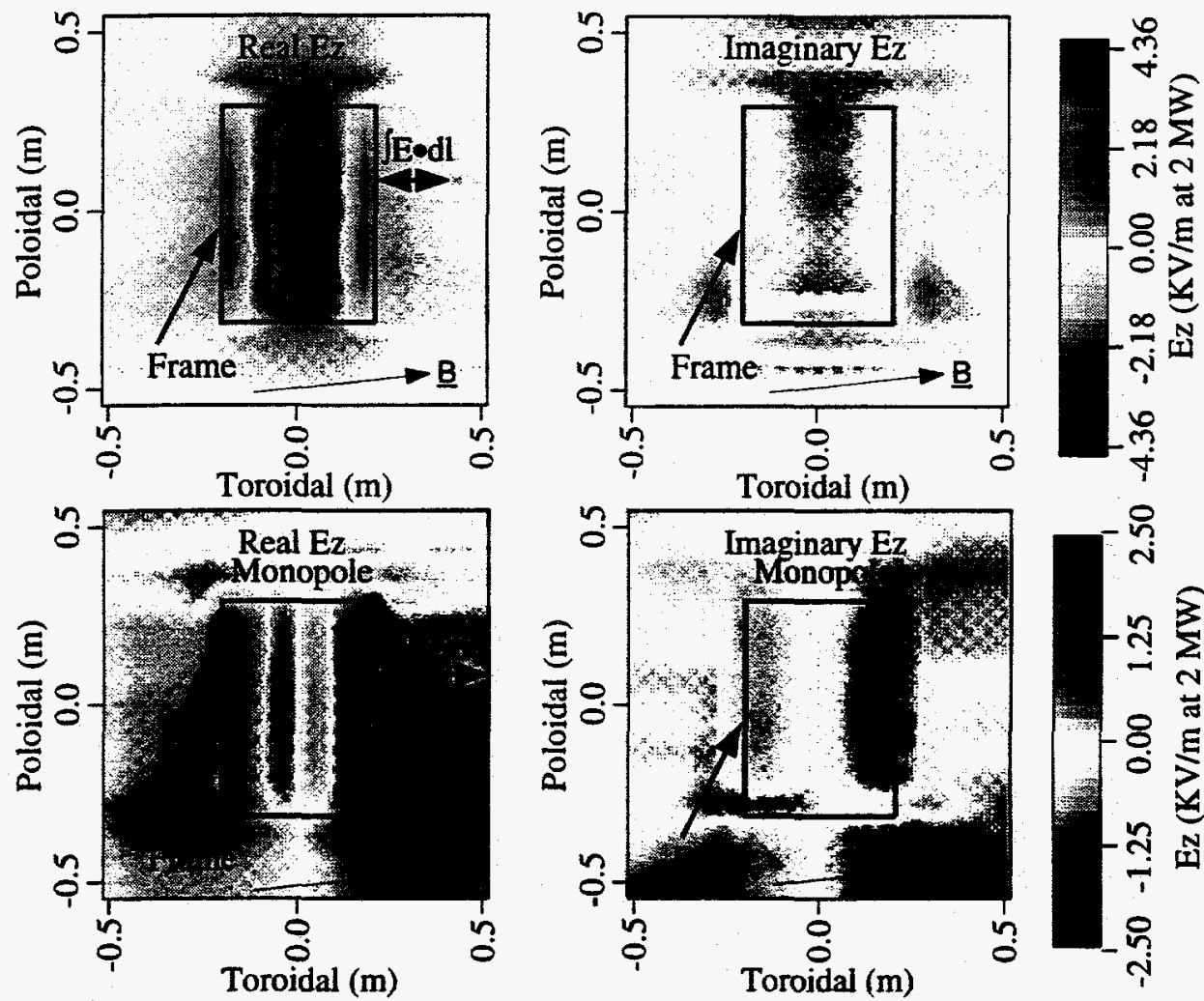

FIGURE 2 The toroidal component of the electric field for dipole and monopole phasing. This field component is primarily caused by charge.

An alternative estimate of the heat flux can be obtained from Fig. 3, which shows the modulus of the parallel electric field accounting for the angle between the static magnetic field and the frame. If the parallel field in Fig. 3 is integrated out to the location where the phase changes by $-\pi / 2$, then $\int \mathrm{E} \cdot \mathrm{dl} \cong 400 \mathrm{~V}$ for dipole phasing, whereas the monopole phasing yields. $\mathrm{J} \cdot \mathrm{dl} \cong 400 \mathrm{~V}$ near the midplane and $\sim 1000 \mathrm{~V}$ near the corners. Such high values for the rectified voltage would not permit RF operation even for dipole phasing which is inconsistent with experimental observations.

Although several assumptions have been made to calculate the rectified voltage, the model seems to provide some connection with realistic experience in Tore Supra. The experiment shows that monopole phasing yields a significantly higher 
heat flux than dipole phasing at the same RF power. The model also shows a left/right asymmetry in the parallel electric fields caused by a combination of the poloidal component in the static magnetic field and the poloidal asymmetry in the plasma response caused by density gradients.
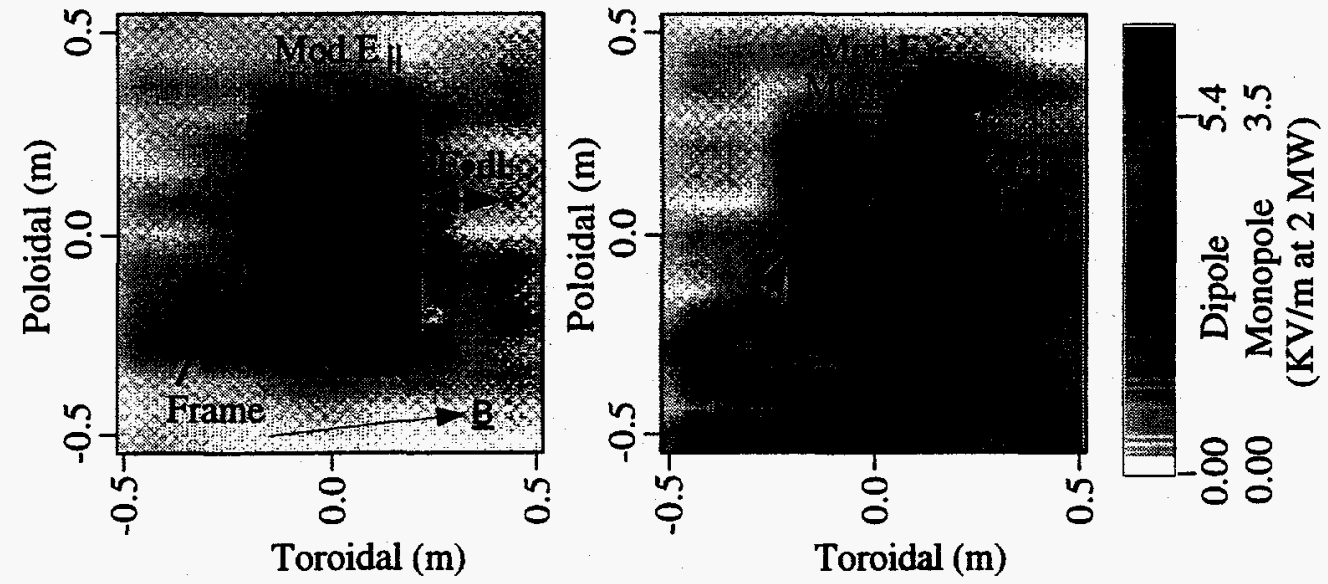

FIGURE 3 Modulus of the parallel electric field for dipole and monopole phasing in Tore Supra. The poloidal electric field component dominates in front of the straps.

\section{SUMMARY}

The RANT3D code has reproduced loading results for several experiments in which good density profile data are available from reflectometry including TFTR and Tore Supra. The code has been used to study the effects of misalignment of the antenna with field lines in Tore Supra. Preliminary results from near-field calculations indicate that the heat flux caused by RF rectification on the outside of the bumper limiters in Tore Supra is on the order of $2 \mathrm{MW} / \mathrm{m}^{2}$ for dipole phasing. The heat flux for monopole phasing may be as much as $10 \mathrm{MW} / \mathrm{m}^{2}$ near the bottom corners of the antenna and $-6 \mathrm{MW} / \mathrm{m}^{2}$ averaged over the limiter. These estimates for the heat flux assume that the electric fields at the bumpers are comparable with the electric fields on the plasma surface $1 \mathrm{~mm}$ away from the bumpers because plasma is not permitted to exist directly on the bumpers in the RANT3D model. A more thorough study of the RF edge problem is required; however, these preliminary results show some consistency with experimental observations.

\section{REFERENCES}

1. Carter, M.D., Rasmussen, D.A., Ryan, P.M., Hanson, G.R., Stallings, D.C., Batchelor, D.B., Bigelow, T.S., England, A.C., Hoffman, D.J., Murakami, M., Wang, C.Y., Wilgen, J., Rogers, J.H., Wilson, J.R., Majeski, R. Schilling, G., to be published in Nucl. Fusion (1995)

2. Brambilla, M., Nucl. Fusion 28, 549 (1988)

3. Stallings, D.C., Batchelor, D.B., Wang, C.Y., Carter, M.D., Bull. Am. Phys. Soc. 39, 1628 (1994)

4. Wang, C.Y., Batchelor, D.B., Jaeger, E.F., Carter, M.D., ORNLTM-12923 (February 1995)

5. Perkins, F.W., Nucl. Fusion 29, 583 (1989)

6. Harris, J.H., Thomas, C.E. Jr., Hogan, J.T., Haste, G.R., Tobin. S., Klepper, C.C., Saoutic, B., Beaumont, B., Becoulet, A., Kuus, H., European Conference on Controlled Fusion and Plasma Physics, Marseilles, 4 (1994)

7. Beaumont, B., Agaraci, G., Gauthier, E., Haste, G., Kuus, H., Schlosser, J., Contributions du CEA au $18^{\text {th }}$ Symposium on Fusion Tech., Karlsruhe, 48 (1994) 